\title{
LOS FINES DE LA EDUCACIÓN DESDE LA PSICOLOGÍA HUMANISTA. UNA MIRADA ONTOLÓGICA
}

The purposes of education from Humanist Psychology. An ontological look

\author{
JOSÉ NAVA BEDOLLA \\ Instituto Superior de Ciencias de la Educación del Estado de México \\ Jose.nava@isceem.edu.mx
}

\section{RESUMEN}

El objetivo central del presente artículo consistió en encontrar la relación entre las necesidades existenciales del sujeto cognoscente y los principios ontológicos del conocimiento, a partir de los cuales se construyen los argumentos sobre los fines de la educación desde la psicología humanista. Se partió del supuesto de que, la propuesta sobre los fines de la educación que se construye desde la psicología humanista, obedece a determinadas necesidades existenciales del sujeto cognoscente. Éstas se encuentran directamente relacionadas con determinados principios ontológicos del conocimiento, dependiendo de circunstancias de modo, tiempo y lugar. A partir del análisis efectuado, se revela la existencia de una relación o correspondencia de dependencia entre las necesidades existenciales del sujeto cognoscente y los principios ontológicos del conocimiento, desde los que se construyen los fines de la educación en la psicología humanista. Entre las conclusiones, se observa que en la teoría psicológica humanista aplicada a la educación, se descuidan los aspectos socioculturales, psicogenéticos, cognoscitivistas, psicoanalistas y conductistas de la educación que son tratados a profundidad por Vygotsky, Piaget, Ausubel, Freud y Skinner, así como por sus seguidores.

Palabras clave: Carl Rogers; filosofía de la educación; fines de la educación; psicología de la educación; psicología humanista; ontología del conocimiento.

\section{ABSTRACT}

The main objective of this article was to find the relationship between the existential needs of the cognoscent subject and the ontological principles of knowledge, from which the arguments on the purposes of education are built, from the perspective of humanist psychology, assuming these are due to certain cognoscent subject's existential needs which are directly related to certain ontological principles of knowledge, depending on 
circumstances of mode, time and place. From the analysis carried out it is revealed the existence of a relationship of dependence or correspondence between the existential needs of the cognoscent subject and the ontological principles of knowledge, from which the purposes of education in humanist psychology are built. Amongst the conclusions, it is observed that the humanist-psychology theory applied to education neglects the sociocultural, psychogenetic, cognitive, psychoanalyst and behavioral aspects of education, which were discussed in depth by Vygotsky, Piaget, Ausubel, Freud and Skinner, as well as by their followers.

Key words: Carl Rogers; philosophy of education; purposes of education; psychology of education; humanist psychology; ontology of knowledge.

\section{INTRODUCCIÓN}

Como resultado de la investigación epistemológica realizada y de los seminarios impartidos sobre la temática a nivel de postgrado en educación en diferentes instituciones públicas y privadas, se ha podido observar algunas relaciones entre las necesidades existenciales de los sujetos cognoscentes y los principios ontológicos del conocimiento. Con conocimiento o no del hecho, dichas relaciones son utilizadas para construir las teorías de la educación, dependiendo de determinadas circunstancias de modo, tiempo y lugar.

Tal es el caso de la psicológica humanista aplicada a la educación. Si se analizan con cuidado los mapeos de este concepto con el término educación elaborados a partir de los supuestos del humanismo psicológico, es posible prestar atención a una serie de relaciones entre las necesidades existenciales del sujeto cognoscente que promueve la teoría y los principios ontológicos del conocimiento. Estos, a su vez, dependen de determinadas circunstancias de la forma, el momento y sitio donde ocurren.

En este artículo se realiza un análisis epistemológico de la noción designada con la expresión fines de la educación desde la mirada de la psicología humanista. Ello, considerando la manera en que se pueden establecer determinadas relaciones entre las necesidades existenciales del sujeto cognoscente y los principios ontológicos del conocimiento que sirven de base para construir los argumentos teóricos.

La pregunta a la cual se busca responder con este análisis es la siguiente: ¿Cuál es la relación entre las necesidades existenciales del sujeto cognoscente y los principios ontológicos del conocimiento que le permiten construir una propuesta sobre los fines de la educación desde la psicología humanista?.

Se parte del supuesto de que los principios ontológicos que se utilizan para elaborar la propuesta sobre los fines de la educación desde la psicología humanista dependen 44 | INTEREDU № 3 VOL. II (DicIEMBRE 2020) PÁGS. 43-59. ISSN: 2735-6523 
directamente de las necesidades existenciales del sujeto cognoscente, dependiendo de circunstancias de modo, tiempo y lugar.

En la primera parte de este análisis se propone que la psicología humanista trata de explicar qué son y cómo se satisfacen las necesidades existenciales de autorrealización individual, tales como: que el ser llegue a ser, el crecimiento espiritual, ético, entre otros. Aplicando lo anterior a la educación, se hace necesario que los fines de ésta sean los de promover la autorrealización de los individuos, estimulando sus potencialidades para que lleguen hasta la altura máxima que la especie humana pueda alcanzar.

Derivado de un análisis epistemológico, en la segunda parte se podrá observar que los principios ontológicos de la psicología humanista aplicada a la educación parten por establecer que las necesidades o fines de la educación son el movimiento o cambio, caos o desorden, indeterminismo o libertad, existencia o presencia, monismo o unidad, entre otras características.

Finalmente, en la tercera y última parte de este ensayo, se plantea lo que parece ser una relación o correspondencia de dependencia entre las necesidades existenciales del sujeto cognoscente y los principios ontológicos del conocimiento desde los que se construyen los fines de la educación a partir de la psicología humanista aplicada a la educación.

Lo anterior orientaría a proponer una relación de causa efecto entre las necesidades existenciales del sujeto cognoscente y los principios ontológicos del conocimiento desde los que se construyen los fines de la educación en la psicología humanista aplicada a la educación, dependiendo de circunstancias de modo, tiempo y lugar. Se tratará de abordar y responder el planteamiento anterior comenzando por proponer una respuesta a la siguiente pregunta: ¿Cuáles son las necesidades existenciales del sujeto cognoscente que le determinan a construir una propuesta sobre los fines de la educación desde la psicología humanista?

\section{LAS NECESIDADES EXISTENCIALES DEL SUJETO COGNOSCENTE EN LA CONSTRUCCIÓN DE LA} PROPUESTA SOBRE LOS FINES DE LA EDUCACIÓN DESDE LA PSICOLOGÍA HUMANISTA

El humanismo o existencialismo “...fue fundado por Abraham Maslow, quien lo concibe como una psicología de ser y no del tener. Esta corriente propone una ciencia del hombre que tome en cuenta la conciencia, la ética, la individualidad y los valores espirituales..." (Guzmán y Hernández, 1993, p. 41). El contenido de esta cita se puede interpretar en el sentido de que a los psicólogos humanistas les interesa el ser y la individualidad de éste. Es decir, la existencia individual del ente que se transforma en ser

por medio de sus vivencias y que se puede expresar mediante el lenguaje. Los 
representantes más importantes de esta teoría con aplicaciones en la sociología, la psicología, la psiquiatría y la educación, son Maslow, Aliport, Rogers, May y Frankl.

En psicoterapia, Rogers (1991, p. 11), adjetiva la corriente humanista como un proceso centrado en la persona: “... No sabría bautizar esta tendencia, pero al pensar en ella la asocio con adjetivos y expresiones tales como fenomenológico, existencial, centrado en la persona; con conceptos como autorrealización, llegar a ser, crecimiento...". Los términos fenomenológico, existencial y centrado en la persona se refieren, respectivamente, a las apariencias, vivencias y lo subjetivo. Además, implican empirismo, realismo y subjetivismo. Las nociones autorrealización, llegar a ser y crecimiento se refieren al indeterminismo, realización y evolución. Todas ellas son nociones que implican movimiento o cambio, caos o desorden, indeterminismo o libertad, existencia o vivencias y unidad o inclusión.

En ese tenor, los psicólogos humanistas se interesan por la autorrealización de la persona, por sus objetivos personales, por su crecimiento o por su desarrollo o evolución personal y espiritual.

El autor, a partir de su práctica psicoterapéutica, expresa:

Es el cliente quien sabe qué es lo que le afecta, hacia dónde se dirige, cuáles son sus problemas fundamentales y cuáles sus experiencias olvidadas. Comprendí que, a menos que yo necesitara demostrar mi propia inteligencia y mis conocimientos, lo mejor sería confiar en la dirección que el cliente mismo imprime al proceso (Rogers, 1991, p. 22).

Es decir, la realización del individuo no debe ser determinada por nadie ni por nada, debe ser una autorrealización. O dicho de otro modo, debe ser una realización indeterminada.

Se estima que sólo las vivencias personales pueden corroborar las creencias de las personas: "Para un nigeriano, una cebra es un animal negro con rayas blancas, mientras que, para un europeo, la cebra es un animal blanco con rayas negras (...) La respuesta es que las experiencias de una persona confirman sus creencias..." (Rogers y Freiberg, 1996, p. 307). Por tanto, todos y cada uno de los individuos sienten y piensan diferente, dependiendo principalmente esta situación de los propios intereses o necesidades existenciales. Por dicha razón, además, es que tienen carencias de carácter desigual.

A partir de lo anterior, cabe plantearse la siguiente pregunta: ¿Cuáles son las necesidades existenciales que el humanismo psicológico aplicado a la educación trata de satisfacer en su propuesta sobre los fines de la educación?

En educación se dice que: "el trabajo del maestro y del educador, como el del terapeuta, está inextricablemente ligado al problema de los valores. La escuela siempre ha 46 | INTEREDU № 3 VOL. II (DiCIEMBRE 2020) PÁGS. 43-59. ISSN: 2735-6523 
sido el medio que utiliza la cultura para transmitir sus valores de una generación a otra..." (Rogers y Freiberg, 1996, p. 15). Es decir, normalmente, la escuela es la que determina los valores de la cultura.

Pero, se supone que los valores se transmiten de un sujeto a otro, cambian con el tiempo, el espacio y las circunstancias:

La cultura del mundo, en todos sus aspectos, es cada vez más científica y relativista, y parecen anacrónicos los rígidos y absolutos valores que heredamos del pasado. Pero quizá lo más importante sea que el individuo está atrapado desde todos los ángulos por demandas de valores divergentes y contradictorios. Ya no es posible, como lo era en un pasado no muy lejano, adoptar cómodamente el sistema de valores de nuestros antepasados, de nuestra comunidad o de nuestra iglesia y vivir la vida sin cuestionarlos o analizar la índole de sus supuestos (Rogers y Freiberg, 1996, p. 316).

Pareciera ser que los valores del pasado están en crisis:

En esta situación no es sorpréndete que el sistema de valores del pasado parezca desintegrarse o destruirse. Los hombres se preguntan dónde están, dónde pueden estar los valores universales. A menudo sentimos que en nuestro mundo moderno no caben los valores generales, básicos para cualquier cultura (Rogers y Freiberg, 1996, p. 316).

No obstante lo anterior, distintas teorías psicológicas aplicadas a la educación están promoviendo formas diferentes de trabajar los valores:

Aunque para la mayor parte de los psicólogos el conductismo ha perdido importancia, éste sigue rigiendo en el sistema educativo del país. Los ejemplos son numerosos: desde la forma en que se impone disciplina a los estudiantes hasta el modo en que se evalúa a los profesores. El método se basa en las ideas básicas de control, premio y castigo, por lo que, dado que escribo en calidad de científico conductista y estoy, al mismo tiempo, profundamente comprometido con todo lo que tiene que ver con lo humano, o personal, lo fenomenológico y lo intangible, me gustaría contribuir en todo lo posible a proseguir con este diálogo en el que tendría que ocupar su lugar central el significado y la posibilidad de libertad, ya que, si consideramos que la enseñanza es un proceso facilitador en el cual se valora al individuo, entonces las palabras libertad y compromiso adquieren pleno sentido (Rogers y Freiberg, 1996, p. 332).

El debate teórico se encuentra trenzado entre el conductismo y el humanismo psicológico. Los primeros son deterministas: “...La mayoría de los científicos de la conducta 
sostienen que el hombre no es libre ni puede comprometerse con algún objetivo como hombre libre, puesto que está condicionado por factores externos a él..." (Rogers y Freiberg, 1996, p. 333). Los segundos son indeterministas:

La libertad de la que hablo es esencialmente interior, existe dentro de la persona aparte de las elecciones y alternativas externas que a menudo pensamos que forman parte de la libertad. Hablo de la clase de libertad que Víktor Frankl describe en su experiencia en el campo de concentración, cuando se despoja a los prisioneros de todo, posesiones, estatus, identidad. Pero los meses y años en tal ambiente demostraron que "se puede despojar a un hombre de todo excepto de una cosa: la última de las libertades humanas, elegir la propia modalidad". 7. V. E. Frankl, From Death Camp to Existencialism (Boston, Beacon Press, 1959) Me he referido a esta libertad interna, subjetiva y existencial. Significa advertir que "puedo vivir yo, aquí, ahora, por mi elección" (Rogers y Freiberg, 1996, p. 340).

El humanismo promueve una autorrealización ética: “...Es el tipo de dirección intencional y significativa que sólo se alcanza gradualmente cuando se está cerca de las propias experiencias, y que respeta tanto las tendencias inconscientes como las elecciones conscientes..." (Rogers y Freiberg, 1996, p. 344).

Se trata de una autorrealización personal: “...el compromiso es la dirección total y cambiante que emerge del individuo, basado en una estrecha y comprensiva relación entre la persona y todas las tendencias de su vida, conscientes e inconscientes..." (Rogers y Freiberg, 1996, p. 345).

Donde el indeterminismo o la libertad de elección genera responsabilidad “...el hombre libre se mueve voluntaria y libre y responsablemente para desempañar su parte significativa en un mundo cuyos acontecimientos determinados se mueven a través de él y a través de su elección y voluntad espontáneas..." (Rogers y Freiberg, 1996, p. 347).

Es una autorrealización en proceso, en constante cambio o movimiento:

Este es mi modelo teórico de las personas que surgen de la terapia o de una situación educativa ideal; la persona que ha experimentado un crecimiento psicológico total, aquella que funciona libremente con todas las potencialidades de su organismo, la que es digna de confianza porque es realista, porque se realiza, porque está socializada y porque se conduce del modo más adecuado; una persona creativa cuya conducta específica no es predecible, que evoluciona constantemente, que se descubre y descubre lo nuevo en sí misma en cada momento (Rogers, y Freiberg, 1996, p. 263).

48 | INTEREDU № 3 VOL. II (DiCIEMBRE 2020) PÁGS. 43-59. ISSN: 2735-6523 
Se puede concluir que la promoción de la autorrealización mediante el estímulo de las potencialidades de los individuos para que lleguen a la altura máxima que la especie humana pueda alcanzar, es la principal necesidad y, por lo mismo, el principal fin que debe promover la educación. Ello, en conformidad con la propuesta de la psicología humanista aplicada a la educación y por medio de las comunidades de aprendizaje: “...Una comunidad de aprendizaje activa no impondrá restricciones sobre las fuentes del aprendizaje: de modo invariable, pondrá en entredicho el qué y el cómo se aprende, aunque esto le lleve a moverse en el filo de la navaja" (Rogers y Freiberg, 1996, p. 399).

De ser así, es pertinente efectuar un análisis que permita dar respuesta a la siguiente pregunta: ¿Cuáles son los principios ontológicos del conocimiento de los que parte la psicología humanista para arribar a la propuesta de los fines de la educación, entendidos como determinadas necesidades de autorrealización?

\section{LOS PRINCIPIOS ONTOLÓGICOS DEL CONOCIMIENTO QUE SE UTILIZAN PARA CONSTRUIR LA} PROPUESTA SOBRE LOS FINES DE LA EDUCACIÓN DESDE LA PSICOLOGÍA HUMANISTA

Buscando comprender los principios del conocimiento, en general, se encontró esta definición del concepto de filosofía de Aristóteles como la “...ciencia teórica de los primeros principios y de las primeras causas..." (1992, p. 8). A primera vista, la definición anterior no dice mucho, pero si se analizan con cuidado los términos utilizados por el filósofo en el predicado de ese juicio, se comprenderá mejor el aserto.

Siguiendo a Aristóteles (1992, p. 75), el concepto expresado como primeros principios se entiende, en un primer momento, como el "...punto de partida de la cosa..."; en segundo lugar, como "...aquello mediante lo que puede hacerse mejor una cosa; por ejemplo, el principio de una ciencia..."; y, finalmente, como “...la parte esencial y primera de donde proviene una cosa".

Si se examinan con cuidado estas tres características de los primeros principios, se puede establecer que la filosofía es un conocimiento hipotético o ciencia teórica, que proporciona los elementos -primeros principios- de donde nacen los saberes con los que se conocen mejor las cosas porque constituyen lo propio, esencial e inicial de las mismas.

Se estima que las exploraciones, descripciones, explicaciones, interpretaciones o comprensiones sobre las cosas, sucesos, entes, objetos o hechos, inician con principios hipotéticos que proporcionan razones primordiales sobre sus fundamentos, orígenes, esencias, causas primeras o raíces verdaderas.

Un principio se puede interpretar como:

La causa fundamental o verdad universal; lo inherente a cualquier cosa. La explicación última del ser de algo. Según Aristóteles, la fuente primaria de todo ser, de toda actualidad y de todo conocimiento. (a) En ontología, los primeros principios INTEREDU № 3 VoL. II (DICIEMBRE 2020) PÁGS. 43-59. ISSN: 2735-6523| 49 
son las categorías o los postulados de la ontología (b) En epistemología, el fundamento de todo conocimiento. Sinónimo frecuente de esencial, universal, causa (Runes, 1998, p. 304).

Se puede decir que un principio constituye el origen, raíz o procedencia primordial, principal o auténtica y, a la vez, universal, esencial e inseparable de la cosa u objeto. Asimismo, que proporciona la explicación última de la existencia de la misma.

En conformidad con la cita anterior, parece ser que existen diferentes tipos de principios: “...puede hablarse de principios del movimiento, o sea, el punto de partida...ontológicos, o sea, de las causas que originan otros entes; $y$, por último, puede hablarse de principios lógicos, que son las razones en las que se apoya cualquier raciocinio..." (Gutiérrez, 2007, p. 157).

Los primeros principios del conocimiento se pueden dividir en: lógicos -las razones en que se apoyan los raciocinios sobre el ser-y ontológicos -las causas del origen del ser-. Para efectos de este trabajo, sólo se abordarán -a manera de ejemplo- algunos de los primeros principios ontológicos.

Los primeros principios ontológicos, se pueden derivar de la suposición que el ser de los entes se manifiesta en la existencia por medio del lenguaje. Para aceptar esto, es necesario recurrir a la definición del concepto de filosofía elaborado por Heidegger (2006, p. 66), la cual se plantea como: “...un corresponder que traduce a lenguaje la llamada del ser del ente...". Si por la expresión ente se entiende todo ser, cosa u objeto que tiene una existencia propia e independiente en la realidad, entonces la enunciación de la categoría nombrada con la expresión filosofía por Heidegger se puede interpretar como la correspondencia que se puede establecer entre el ente -como posibilidad de existencia-y el ser-como experienciaa través del lenguaje. Es decir, como la manifestación del ser del ente mediante expresiones, frases, palabras, locuciones o voces; o sea, por medio de la comunicación.

Algunos principios ontológicos del conocimiento son los siguientes:

\subsection{CAMBIO O MOVIMIENTO.}

Heráclito es el primer pensador para el cual la esencia del mundo consiste en que se mueve. Para él, toda materia se resuelve en aquello que pasa con ella. En el lugar del ser definido y determinado coloca la eterna sucesión de los contrarios en que la muerte de una forma se convierte en la vida de otra. Y lo mismo que a todo lo particular, concibe el mundo como conjunto en un constante devenir y pasar (Simmel, 1946, p. 77). 
Se supone que la realidad se encuentra en constante cambio, permuta, canje, movimiento, agitación, sacudiéndose, revuelta, meneándose, deviniendo, sucediendo, aconteciendo, pasando, acaeciendo, ocurriendo. Toda palabra o término que indique la idea de movimiento o cambio, permite suponer que la realidad nunca está quieta, inmóvil. Lo contrario del cambio o movimiento lo es el reposo o la quietud.

En la proposición de Protágoras de que "el hombre es la medida de todas las cosas, de las que existen porque existen, de las que no existen porque no existen..." (Simmel, 1946, p. 106), se puede decir que todos y cada uno de los seres humanos sentimos y pensamos de manera diferente. Es decir, desde este principio es posible conjeturar que la realidad cambia o se mueve de un sujeto cognoscente a otro.

\subsection{CAOS O DESORDEN.}

Bacon (1991, p. 39) parte de la realidad para remontarse a los principios; es decir, del caos al orden: “...para penetrar en las entrañas de la naturaleza, es preciso que, tanto las nociones como los principios, sean sacados de la realidad...". Se sospecha que el contexto se encuentra en constante desorden o caos. Toda palabra o término que indique nociones de desconcierto, anarquía o desorganización permite admitir que en el ambiente impera el caos o desorden.

Se puede observar de la siguiente manera desde la perspectiva de Bacon (1991, p. 39):

$\mathrm{Ni}$ hay, ni puede haber más que dos vías para la investigación y descubrimiento de la verdad: una que, partiendo de la experiencia y de los hechos, se remonta enseguida a los principios que adquieren una autoridad incuestionable, juzga y establece las leyes secundarias (axiomas medios) ...y otra que, de la experiencia y de los hechos induce leyes, elevándose progresivamente sin sacudidas hasta los principios más generales que alcanza en último término.

Es decir, la verdad se induce, partiendo de las experiencias y los hechos hacia los principios. Se trata del método inductivo.

\subsection{INDETERMINISMO O INDEFINICIÓN.}

Para Locke (1994, p. 321), la realidad consiste en momentos infinitos de experiencias: “...si nuestras débiles facultades no son capaces de separar la sucesión de cualquier tipo de duración, nuestra idea de la eternidad no puede ser más que una sucesión infinita de momentos de la duración en la cual algo existe...". Es decir, se conjetura que los ambientes están indeterminados, indefinidos, vacilantes, inciertos e incluso desconocidos.

Considerando detenidamente el juicio que se menciona, es posible observar que deriva de un principio ontológico de indeterminación del ser del ente. Es decir, se presume 
que los entornos de encuentran en estado de indeterminación, irresolución, incertidumbre, relatividad y perplejidad.

\subsection{VIVENCIA O EXISTENCIA}

Para Berkeley (citado por Cassirer II, 1986, p. 253) la realidad existe con independencia de que haya o no un sujeto que la pueda percibir: “...los árboles son en el jardín, están en él, quiéralo yo o no lo quiera, ya me los representé o no; pero esto sólo quiere decir una cosa: que no tengo más que ir al jardín y abrir los ojos, para verlos necesariamente...". Se estima que el entorno o realidad existe o vive.

Todo término o palabra que indique nociones como vida, subsistencia, realidad, esencia, sustancia, ser, haber, entre otras, permite aceptar que el contexto o realidad tiene existencia o vivencia.

\subsection{UNIDAD O INCLUSIÓN.}

Los primeros principios ontológicos mencionados en párrafos anteriores, tales como: cambio o movimiento, caos o desorden, indeterminismo o indefinición, vivencia o existencia, permiten entender el contexto o realidad como un todo o concierto de eventos interrelacionados los unos con los otros.

Todo término o palabra que indique ideas de singularidad, individualidad, individuo, indivisibilidad, uno, entre otras cosas, permite presumir que la situación o realidad de que trata constituye una unidad o inclusión.

Una vez analizados los principios ontológicos presentados, cabe preguntarse ¿Cuál es la relación entre las necesidades existenciales del sujeto cognoscente y los principios ontológicos del conocimiento que permiten construir una propuesta sobre los fines de la educación en términos de promover la autorrealización del individuo, estimulando sus potencialidades para que llegue hasta la altura máxima que la especie humana puede alcanzar, desde la psicología humanista aplicada a la educación?

3. LAS NECESIDADES EXISTENCIALES DEL SUJETO COGNOSCENTE Y LOS PRINCIPIOS ONTOLÓGICOS DEL CONOCIMIENTO EN LA CONSTRUCCIÓN DE LA PROPUESTA DE LA PSICOLOGÍA HUMANISTA SOBRE LOS FINES DE LA EDUCACIÓN

3.1 EL PRINCIPIO ONTOLÓGICO DE MOVIMIENTO O CAMBIO Y LOS FINES DE LA EDUCACIÓN QUE SE PROMUEVEN DESDE LA PSICOLOGÍA HUMANISTA

Rogers y sus seguidores son de la opinión de que los fines de la educación, es decir, sus necesidades existenciales, son la promoción de la autorrealización, estimulando las 52 | INTEREDU № 3 VOL. II (DiCIEMBRE 2020) PÁGS. 43-59. ISSN: 2735-6523 
potencialidades de los educandos. Ello, para que lleguen hasta la altura máxima que la especie humana puede alcanzar. Para su alcance, una de las condiciones por las que se debe comenzar es aceptar que todos los seres humanos sentimos y pensamos de manera diferente a los demás: “...he aprendido que toda vez que una persona se mostró deseosa de comunicarme algo acerca de sus tendencias internas, esto me ha resultado útil, aunque sólo fuese para advertir que las mías son diferentes..." (Rogers, 1991, p. 26). Para arribar a la autorrealización individual mediante el estímulo de las potencialidades para que lleguen hasta la altura máxima que la especie humana puede alcanzar, se supone el cambio o movimiento de la realidad educativa de un sujeto a otro y de cada sujeto en sí mismo, dependiendo de circunstancias de modo, tiempo y lugar en que dicho sujeto se ubique. Es decir, se debe considerar que todos y cada uno de los sujetos del acto educativo siente y piensa diferente de los demás y de sí mismos en las distintas circunstancias de modo, tiempo y lugar en que se ubiquen.

Si fijamos la atención en el término señalado en cursivas en la cita anterior, se podrá observar que se refiere a que las tendencias internas de todos y cada uno de los sujetos se ubican en lo desigual, en los otros, las otras, los disímiles. Es decir, al movimiento, al cambio de las tendencias internas de un sujeto a otro.

Algo parecido se podrá notar en la referencia siguiente: “...Los clientes parecen convertirse cada vez más abiertamente en un proceso de constante cambio y adquieren mayor fluidez..." (Rogers, 1999, p. 156). Los términos indicados con cursivas se refieren a permutas o canjes. Es decir, los sentidos y significados de estas palabras se pueden interpretar como que los clientes están en constante movimiento o cambio tanto entre ellos mismos como entre unos y otros. El término movimiento también se puede apreciar en la siguiente cita:

Pienso que los clientes cuya terapia ha acusado movimientos significativos viven más intensamente sus sentimientos de dolor, pero también disfrutan más de sus satisfacciones; sienten el odio con mayor claridad, pero también el amor, el miedo es una experiencia que conocen más profundamente, pero también lo es el coraje. La razón por la que pueden llevar una vida plena reside en la confianza que poseen en sí mismos como instrumentos idóneos para enfrentar la vida (Rogers, 1999, p. 175).

Aplicando las ideas anteriores al plano educativo, dicho concepto se puede entender en términos de corrientes o tendencias. En otras palabras, como los cambios que se han operado en los alumnos a partir de las clases centradas en la psicología humanista.

Rogers y Freiberg (1996, p. 91) señalan también que encontrarse a sí mismo es un proceso que dura toda la vida: “El proceso de encontrarse a sí mismo, de aceptarse y de mostrarse como es, no es algo que sólo tenga lugar en la terapia o en los grupos (...) la búsqueda por llegar a ser la persona que de manera tan singular somos, es un proceso que dura toda la vida". 
Las palabras que indican cambio, sentidos y significados de movimiento, permiten explicar el aprendizaje como proceso:

"Creo que nos enfrentamos a una situación enteramente nueva en educación, en la cual el propósito de ésta, si hemos de sobrevivir, debe ser la facilitación de cambio y el aprendizaje. Solo son educadas las personas que han aprendido cómo aprender, que han aprendido a adaptarse y a cambiar, que advirtieron que ningún conocimiento es firme, que sólo el proceso de buscar el conocimiento es una base para la seguridad. El único propósito válido para la educación en el mundo moderno es el cambio y la confianza en el proceso y no en el conocimiento estático" (Rogers y Freiberg, 1996, p. 184).

Los argumentos que se analizan, privilegian el cambio y el movimiento en contra de la quietud o lo estático, lo que no se mueve, lo que no cambia.

Concluyendo: “Para Rogers (citado por Patterson, 1986); la educación tiene el importante papel de enseñar a las personas a vivir en paz, evitando las guerras, adiestrarlas en el cuidado del medio ambiente y sobre todo ayudar a los individuos a vivir en un mundo en perpetuo cambio, donde lo más importante no es adquirir conocimientos sino aprender a aprender" (Guzmán y Hernández, 1993, p. 45).

\subsection{EL PRINCIPIO ONTOLÓGICO DE CAOS O DESORDEN Y LOS FINES DE LA EDUCACIÓN QUE} SE PROMUEVEN DESDE LA PSICOLOGÍA HUMANISTA

Si suponemos que los fines de la educación se logran estimando que la misma se encuentra en constante cambio o movimiento de un sujeto a otro como de todos y cada uno de los sujetos en sí mismos, dependiendo de circunstancias de modo, tiempo y lugar; entonces también, y de manera necesaria debemos opinar que la educación se encuentra en un completo estado de caos o desorden.

Rogers y sus seguidores se refieren a los fines de la educación suponiendo -a causa de determinados principios ontológicos de los que parten para generar nuevos conocimientosque en la misma impera el caos o desorden: “...El auténtico sí mismo se descubre naturalmente en las experiencias propias, sin pretender imponerse a ellas" (Rogers, 1999, p. 109). El término en cursivas indica prácticas, usos, usanzas, rutinas y estilos diferentes. Es decir, los sí mismos cambian o se mueven de un sujeto a otro. Los sujetos no deben imponerse sobre sus experiencias, sino dejar que fluyan. No deben permitir ser determinados por ellas ni por las experiencias de otros. En los sí mismos debe reinar el caos, el desconcierto, la anarquía, la desorganización, el desorden, el desbarajuste, el desarreglo. 
Si estimamos que la educación cambia o se mueve de un sujeto a otro y de un sujeto en sí mismo, dependiendo de circunstancias de modo, tiempo y lugar, es porque también opinamos que se encuentra en completo caos y desorden.

Se argumenta que, para ser una persona completa, todo sujeto debe experimentar, ser libre, espontáneo y hacer su voluntad: “...la persona que funciona de modo integral no sólo experimenta, sino que también hace uso de la más absoluta libertad cuando elige de manera espontánea y voluntaria aquello que, por otra parte, también está absolutamente determinado" (Rogers, 1999, p. 173). Las palabras escritas en cursivas indican autonomía, liberación, franqueza, apertura, discrecionalidad y potestad. Se estima que, para lograr los fines de la educación, entre los que se encuentra la formación de sujetos íntegros, se debe suponer necesariamente el caos o el desorden.

La psicoterapia centrada en el sujeto, que después será recuperada en la teoría educativa por este autor, promueve que “...los clientes de este tipo de psicoterapia -se refieren a la psicoterapia centrada en el cliente- tienden a apoyarse menos en los valores y expectativas de los otros y comienzan a confiar más en las evaluaciones de sí mismos basadas en sus propias experiencias" (Rogers, 1999, p. 222). Es decir, todo sujeto debe ser autónomo en sus decisiones, para llegar a la autorrealización: “...La autorrealización y la tolerancia son virtudes que toda buena educación debería promover..." (Guzmán y Hernández, 1993, 45).

\subsection{EL PRINCIPIO ONTOLÓGICO DE INDETERMINISMO O LIBERTAD Y LOS FINES DE LA} EDUCACIÓN QUE SE PROMUEVEN DESDE LA PSICOLOGÍA HUMANISTA

Si conjeturamos que los fines de la educación se consiguen apreciando que ella misma se encuentra en firme cambio o movimiento, desorden o caos, de un sujeto a otro y de todos y cada uno de los sujetos en sí mismos, dependiendo de circunstancias de modo, tiempo y

lugar; entonces también y de manera necesaria, debemos opinar que los sujetos del acto educativo se encuentran en estado de indeterminación o incertidumbre.

Rogers y sus seguidores se refieren a los fines de la educación considerando que los sujetos del acto educativo deben ser comprendidos, debido a determinados principios ontológicos de indeterminismo: “...He descubierto el enorme valor de permitirme comprender a otra persona..." (Rogers, 1991, p. 28). Interpretando el término anterior en sus sentidos y significados de ponerse en el lugar del otro, respetar su libertad, espontaneidad y voluntariedad.

Libertad, independencia, espontaneidad, ser franco, abierto, directo, ingenuo y voluntarista, ser discrecional, facultativo, potestativo y deliberado son consideradas las bases para la autorrealización: “Aparentemente, el objetivo más deseable para el individuo, 
la meta que persigue a sabiendas o inconscientemente, es llegar a ser él mismo..." (Rogers, 1999, p. 104).

Maslow (1988, citado por Guzmán, 1993, p. 45), se refiere a los fines de la educación de la siguiente manera:

La función última de la educación es desde esta postura la de promover la autorrealización; o sea, la educación debe estimular las potencialidades de los individuos para que lleguen hasta la altura máxima que la especie humana puede alcanzar. El propósito final de la educación es ayudar a que las personas logren lo mejor de lo que son capaces.

Es decir, una de las condiciones necesarias para arribar a la autorrealización y estimular las potencialidades para que los educandos lleguen hasta la altura máxima que la especie humana puede alcanzar consiste en que se encuentren en un estado invariable de indeterminación.

\subsection{EL PRINCIPIO ONTOLÓGICO DE EXISTENCIA, PRESENCIA O IMPACTO Y LOS FINES DE LA EDUCACIÓN} QUE SE PROMUEVEN DESDE LA PSICOLOGÍA HUMANISTA

Carl R. Rogers y sus seguidores se refieren a los fines de la educación estimando debido a determinados principios ontológicos de existencia, presencia o impacto en la realidad educativa de los que, con conocimiento o no del hecho, parten para hacer ciencia de la conducta- que: “...la mayor parte de los casos en que no logro (sic) ser útil a otros individuos pueden explicarse por el hecho de que, a causa de una actitud defensiva, me comporto de una manera superficial y opuesta a mis verdaderos sentimientos" (Rogers, 1991, p. 27). Ser real significa existir, ser serio e histórico. Es decir, impactar los sentidos de los demás, aceptarse tal y como uno es:

Otro resultado que parece surgir del hecho de aceptarse tal como uno es consiste en que sólo entonces las relaciones se tornan reales. Las relaciones reales son atractivas por ser vitales y significativas (...) Las relaciones reales no permanecen estáticas, sino que tienden a ser cambiantes (Rogers, 1991, p. 27).

Es decir, las relaciones reales o existentes se mueven, cambian. En ellas reina el caos o el desorden. Además, son indeterminadas e impactan los sentidos de quienes nos rodean. Es decir, existen. Todo esto refiere a que los seres humanos son indeterminables. En otras palabras, obran a partir de sus necesidades existenciales y éstas se encuentran en constante cambio. 
En el campo de la psicoterapia: “Otra tendencia de este tipo se manifiesta en el hecho de que el cliente se aleja de la imagen compulsiva de lo que debería ser..." (Rogers, 1999, p. 153). Lo mismo sucede con la educación. El sujeto real, aquel del acto educativo se ubica en el ser, alejándose del deber ser. Por el término ser se entiende al ente, individuo, espécimen, la existencia, la entelequia. Por tanto, es lo que existe, lo que está, lo que florece, lo que coexiste; en otras palabras, existir es impactar a los demás y ser impactado por éstos: “...Lo que soy y lo que siento es suficientemente bueno como para servir de base a una terapia, siempre que logre ser lo que soy y lo que siento en mi relación con él. Entonces él también logre ser lo que es, de manera abierta y libre de temor" (Rogers, 1999, p. 69).

“Los humanistas conciben la universidad ideal, como el espacio donde los estudiantes acudirán por iniciativa propia a cursar las asignaturas que respondan a sus inquietudes personales de aprendizaje, sin que estuvieran prestablecidos créditos ni grados o cursos obligatorios..." (Guzmán, 1993, pp. 45-46).

Esta es otra de las condiciones necesarias para arribar a la autorrealización y estimular las potencialidades para que los educandos lleguen hasta la altura máxima que la especie humana puede alcanzar, consiste en ser auténtico, fidedigno y puro. En otras palabras, ser real.

\subsection{EL PRINCIPIO ONTOLÓGICO DE UNIDAD O TOTALIDAD Y LOS FINES DE LA EDUCACIÓN} QUE SE PROMUEVEN DESDE LA PSICOLOGÍA HUMANISTA

Rogers (1999, p. 67) estima que mediante un tratamiento psicoterapéutico:

El individuo se acerca paulatinamente a la fluidez y adquiere la capacidad de cambiar, reconocer y aceptar sus sentimientos y experiencias, formular constructos provisionales, descubrirse en su propia experiencia como una persona cambiante, $\mathrm{y}$ establecer relaciones auténticas y estrechas; en fin, se convierte en una unidad y alcanza la integración de sus funciones.

La palabra unidad se entiende como un dispositivo, aparato, mecanismo, módulo o componente. Es decir, lo integrado que, a su vez, significa completo y acabado, único e insuperable. Todos y cada uno de los individuos que habitamos este mundo somos únicos e irrepetibles. El término integración se puede interpretar como composición o combinación de las funciones del individuo.

Por ello, “...a los humanistas no les interesa tanto la naturaleza y validez del conocimiento en sí, como la aplicación de nuevos procedimientos metodológicos que enriquezcan la parte crucial del conocimiento: la comprensión del hombre como persona total" (Guzmán y Hernández, 1993, p. 45). El término total se puede interpretar como INTEREDU № 3 VoL. II (DICIEMBRe 2020) PÁGS. 43-59. ISSN: 2735-6523| 57 
sistémico e integral. Es entonces, cuando los fines o necesidades existenciales de la educación, no pueden ser otros más que promover la autorrealización del individuo particular estimulando sus potencialidades para que lleguen al mayor desarrollo que la especie humana pueda conseguir.

\section{CONCLUSIONES}

La pregunta guía del presente ensayo fue la siguiente: ¿Cuál es la relación entre las necesidades existenciales del sujeto cognoscente y los principios ontológicos del conocimiento que le permiten construir una propuesta sobre los fines de la educación desde la psicología humanista?

La respuesta a dicha cuestión se determinó en el sentido de que la promoción de la autorrealización de los estudiantes mediante el estímulo de sus potencialidades para el alcance de su máximo desarrollo -es decir, las necesidades existenciales o fines de la educación-, sólo es posible partiendo de principios ontológicos que supongan movimiento o cambio, caos o desorden, indeterminismo o libertad, existencia, presencia o impacto, unidad o totalidad. En otras palabras, este análisis permitió determinar que existe una relación de causa efecto entre las necesidades existenciales del sujeto cognoscente y los principios ontológicos del conocimiento que le permiten construir una propuesta sobre los fines de la educación desde la psicología humanista aplicada a la educación. Se partió del supuesto de que la propuesta sobre los fines de la educación que se construye desde la psicología humanista obedece a determinadas necesidades existenciales del sujeto cognoscente que se encuentran directamente relacionadas con determinados principios ontológicos del conocimiento, dependiendo de circunstancias de modo, tiempo y lugar.

Por tanto, el objetivo central del análisis efectuado consistió en encontrar la relación entre las necesidades existenciales del sujeto cognoscente (la autorrealización de los estudiantes mediante el estímulo de sus potencialidades para que lleguen hasta la altura máxima que la especie humana puede alcanzar) y los principios ontológicos del conocimiento (movimiento o cambio, caos o desorden, indeterminismo o libertad, existencia, presencia o impacto, unidad o totalidad, y demás), a partir de los cuales se construyen los argumentos sobre los fines de la educación desde la psicología humanista.

Dicho objetivo se cumplió en el sentido de que es posible explorar, describir, explicar, interpretar o comprender la relación entre las necesidades existenciales del sujeto cognoscente (la autorrealización de los estudiantes mediante el estímulo de sus potencialidades para que lleguen hasta la altura máxima que la especie humana puede alcanzar) y los principios ontológicos del conocimiento (movimiento o cambio, 
caos o desorden, indeterminismo o libertad, existencia, presencia o impacto, unidad o totalidad, y demás).

Una lectura detallada de las necesidades existenciales o de los fines de la educación desde el humanismo, en relación con algunos principios ontológicos del conocimiento, permite inferir que entre los dos se puede observar una relación de causa-efecto.

La promoción de la autorrealización de los estudiantes mediante el estímulo de sus potencialidades para que lleguen hasta la altura máxima que la especie humana puede alcanzar, (es decir, las necesidades existenciales o fines de la educación) sólo es posible partiendo de principios ontológicos que supongan movimiento o cambio, caos o desorden, indeterminismo o libertad, existencia, presencia o impacto, unidad o totalidad, y demás.

La autorrealización del espíritu humano es muy deseable en el contexto actual de la educación. No obstante, se descuidan los aspectos socioculturales, psicogenéticos, cognoscitivistas, psicoanalistas y conductistas de la educación que son tratados a profundidad por Vygotsky, Piaget, Ausubel, Freud y Skinner, y sus seguidores.

\section{REFERENCIAS BIBLIOGRÁFICAS}

Aristóteles (1992). Metafísica. México. Porrúa (Col. “Sepan Cuántos...”, núm. 120).

Bacon, Francisco (1991). Novum organum. México. Porrúa (Col. "Sepan cuantos...”, núm. 293).

Cassirer, Ernest (1986). El problema del conocimiento en la filosofía y en la ciencia moderna I. México. Editorial Fondo de Cultura Económica.

(19xx). El problema del conocimiento en la filosofía y en la ciencia modernas II. México. Editorial Fondo de Cultura Económica.

Gutiérrez Sáenz, Raúl (2005). Introducción a la lógica. México. ESFINGE.

Guzmán, Jesús Carlos \& Gerardo Hernández Rojas (1993). Implicaciones educativas de seis teorías psicológicas. México. CONALTE.

Heidegger, Martín (2006). ¿Qué es la filosofía? España. Herder.

Locke, John (1994). Ensayo Sobre el Entendimiento Humano. Tomo I. México. Gernika.

Rogers, Carl (1991). El proceso de convertirse en persona. México. Editorial Paidós Mexicana, S. A. R. Rogers, Carl \& H. Jerome Freiberg (1996). Libertad y creatividad en la educación. España. Paidós Educador.

Runes, Dagoberto D. (1998). Diccionario de filosofía. México. Grijalbo.

Simmel, Jorge (1946). Problemas fundamentales de la filosofía. Madrid. Revista de Occidente. 